\title{
Forschendes Lernen als Studiengangsprofil - was meint dies konkret?
}

\section{Margrit E. Kaufmann und Henning Koch}

Dieser Beitrag zeigt beispielhaft auf, wie an der Universität Bremen das Forschende Lernen als Studiengangsprofil implementiert wird. Dabei wird Bezug genommen auf eine Begleitforschung zum studentischen Erleben des Forschenden Lernens, sowohl im Einzelmodul als auch im Verlauf des Curriculums. Auch wird nachgezeichnet, wie sich - basierend auf den Ergebnissen der Begleitforschung - im Lehrteam an der curricularen Profilbildung zum Forschenden Lernen arbeiten lässt. Abschließend wird nach der Übertragbarkeit in andere Kontexte gefragt.

\section{$1 \quad$ Einleitung}

An der Universität Bremen baut die Profilbildung zum Forschenden Lernen auf der Tradition des Projektstudiums auf, das als „Bremer Modell“ bekannt ist (Huber, Kröger \& Schelhowe, 2013). Forschendes Lernen wurde, mit Partizipation und Vielfalt, 2015 im Leitbild von Studium und Lehre verankert (Universität Bremen, 2015) und ist auch in der Universitätsstrategie prominent vertreten (Strategiedokument Universität Bremen, 2018). Besonders vorangetrieben wird die Profilbildung zum Forschenden Lernen durch das im Rahmen des Qualitätspakts Lehre geförderte Projekt „Forschend studieren von Anfang an - Heterogenität als Potenzial“, kurz 
„ForstA“, das derzeit mit „ForstAintegriert“ fortgesetzt wird. Dieses Projekt verfolgt das Ziel, Forschendes Lernen als Profil von ganzen Studiengängen zu etablieren.

$\mathrm{Zu}$ der Frage, wie diese Profilbildung in einem einzelnen Studiengang gelingen kann, führen wir am Institut für Ethnologie und Kulturwissenschaft mit dem Bachelorstudiengang Kulturwissenschaft ein Pilotprojekt durch, das auf einem Begleitforschungsprojekt mit Studierenden, der Zusammenarbeit am Curriculum mit den Lehrenden und begleitenden Maßnahmen zur Förderung Forschenden Lernens basiert. Den Anstoß dazu gab der durch unsere Evaluationen verdeutlichte Bedarf, das Curriculum als Ganzes unter der Perspektive des Forschenden Lernens in den Blick zu nehmen, um dabei die einzelnen Module besser miteinander zu verknüpfen. Als grundlegend für das Forschende Lernen erachten wir, aufbauend auf unseren Evaluations- und Begleitforschungen, die Kommunikation unter und zwischen den Lehrenden und den Studierenden zu stärken und fruchtbar zu machen (Kaufmann \& Koch, 2015a, 2015b; Ghaffarizad et al., 2015). Beim Forschenden Lernen setzen wir an den Fachkulturen, den langjährigen Erfahrungen im Rahmen von Lehrforschungsprojekten und am Bremer Projektstudium an (Kaufmann, 2013; Kaufmann \& Schelhowe, 2017). Der Erhalt dieser Traditionen des Projektstudiums und der Lehrforschungsprojekte wurde durch die Modularisierung erschwert. Über die Projekte zum Forschenden Lernen knüpfen wir daran an und versuchen dabei, durch die Arbeit mit den Lehrenden und Studierenden fachkulturelle Besonderheiten zu explizieren und zu stärken.

In diesem Beitrag stellen wir nach einer kurzen Beschreibung wesentlicher Momente der Profilbildung zum Forschenden Lernen an der Universität Bremen die zentralen Ebenen unseres Projekts zur strukturellen Verankerung Forschenden Lernens am Beispiel eines einzelnen Studiengangs vor. Wir zeigen zum einen, wie wir die Begleitforschung mit den Studierenden angelegt und durchgeführt haben. Zum anderen gehen wir auf die Arbeit mit den Lehrenden an den intermodularen Verbindungen und am Studiengangsprofil ein. Schließlich heben wir die Ebene der Kollaboration und das Eingehen auf die Fachspezifika bei der Implementierung des Forschenden Lernens besonders hervor.

\section{Studiengangsprofilbildung an der Universität Bremen}

Bei der Profilbildung zum Forschenden Lernen an der Universität Bremen liegt die Hauptverantwortung für die Umsetzung bei den einzelnen Fachbereichen, vor allem aber bei den Personen, welche die Studiengänge konzeptionell planen. Ein solcher Prozess der Herausbildung eines Lehrprofils für eine ganze Universität, an dem 
sich möglichst viele beteiligen, ist schrittweise durchzuführen und recht aufwendig (Kaufmann \& Schelhowe, 2017). Ein Kreis von internen und externen Fachleuten für Forschendes Lernen unterstützt die Universitätsleitung dabei, Qualitätskriterien festzulegen und geeignete Anträge für die Umsetzung auszuwählen.

An der Universität Bremen lag der Fokus der Profilbildung zuerst, gemäß ihrer Tradition des Projektstudiums, auf einzelnen Projekten oder Lehrveranstaltungen und auf der Arbeit an einem geteilten Verständnis des Forschenden Lernens. Dies erfolgte nach dem Ansatz Ludwig Hubers (Huber, Kröger \& Schelhowe, 2013), wonach den Studierenden, meist in einem Modul, das Erleben des ganzen Bogens des Forschens ermöglicht wird - von der Entwicklung einer Fragestellung bis hin zu der Veröffentlichung der Forschungsergebnisse. In der Folge geht es nun, angelehnt an das „Zürcher Framework“ (Tremp \& Hildbrand, 2012), um den Schritt vom Einzelmodul zur curricularen Profilbildung (Kaufmann \& Koch, 2018). Nach dem Framework lassen sich einzelne Schritte des Forschenden Lernens im gesamten Curriculum verdeutlichen und im Zusammenhang betrachten. Es orientiert sich an den studentischen Lern- und Forschungsaktivitäten als verschiedenen, zusammenhängenden Etappen eines mehrjährigen Forschungsprozesses.

Inzwischen arbeiten einige Studiengänge an der Profilbildung nach diesem Modell. Für die Implementierung des Profils und zur besseren Gestaltung von Lehre und Studium sind viele Kanäle und Plattformen der Kommunikation zwischen der Leitungsebene, der Fachbereichs- und Studiengangsebene sowie der Modul- und Veranstaltungsebene nötig. Auf der Leitungsebene gibt die Universität Bremen beispielsweise eine Zeitschrift für Lehre heraus („Resonanz - Magazin für Lehre und Studium an der Universität Bremen“), über die ein reger Austausch zum Forschenden Lernen stattfindet. Darin schreiben Lehrende und Studierende. Zudem hat der Konrektor für Lehre und Studium eine „Standing Conference für Innovation in der Lehre" eingerichtet, um den inneruniversitären Austausch zu guter Lehre zu fördern. Am jährlich stattfindenden „Tag der Lehre“ finden sowohl in den Fächern Aktivitäten von Lehrenden und Studierenden statt als auch zentrale universitätsweite Veranstaltungen. Außerdem wird ein Preis für gute Lehre verliehen. Auch unter den Pilotprojekten war der regelmäßige Austausch von Bedeutung, der durch das Referat für Lehre und Studium koordiniert wurde. Im Verlauf entwickelte sich auch die gemeinsame Projektmaßnahme „Exploring Diversity!“ (Satilmis \& Voss, 2017). Dabei haben zwei der Pilotstudiengänge die Studierenden, die als Coaches oder Tutorinnen und Tutoren andere Studierende in den Prozessen des Forschenden Lernens begleiten, gemeinsam zum Umgang mit Diversität beim Forschenden Lernen geschult (zum Thema Diversität im Forschenden Lernen siehe auch den Beitrag von Satilimis in diesem Sammelband). 


\section{Das Projekt „Forschendes Lernen als Studiengangsprofil im BA Kulturwissenschaft"}

Unser Projekt „Forschendes Lernen als Studiengangsprofil im BA Kulturwissenschaft" ist eines der drei Pilotprojekte zur Profilbildung im Sinne des Forschenden Lernens. Als Best-Practice-Beispiel standen wir damit vor der Aufgabe, das Forschende Lernen als Profil in unserem Studiengang zu systematisieren. Dabei ist herauszustellen, dass die Arbeit am Forschenden Lernen mit dem Start des Projektes keinesfalls bei Null anfing, sondern an eine lange Tradition des Forschens im Studium anknüpfte. Dies gründet auf dem wissenschaftstheoretischen und methodologischen Selbstverständnis der Ethnologie und Kulturwissenschaft. In diesen Fächern sind Theorie und Empirie, beispielsweise Ethnologie und Ethnografie, besonders eng verbunden. Somit lernen die Studierenden unseres Fachs seit jeher, sich einer Fragestellung mittels eigener Forschungsaktivitäten, bei denen sie Theorien und Praxis verbinden, anzunähern. Völlig neu ist jedoch die Herausforderung, das Forschende Lernen und einzelne Forschungsprozessschritte systematisch zur Strukturierung des gesamten Studienverlaufs heranzuziehen. Doch konnten wir uns auch dabei auf Projektergebnisse rückbeziehen, die wir im Rahmen des Projekts „Forschend studieren von Anfang an - Heterogenität als Potenzial“ speziell in Bezug auf die Studieneingangsphase generiert hatten. Zentrales Ergebnis dieses Projektes war, dass das Konzept des Forschenden Lernens aufseiten der Studierenden nur fruchtbar werden kann, wenn sie ausreichend darüber und über die Sinnhaftigkeit des Aufbaus ihres Studiums und die Verbindungen zwischen den Modulen aufgeklärt werden. Forschendes Lernen als Studiengangsprofil zu implementieren gestaltet sich deshalb von Beginn an als Kommunikations- und Vermittlungsaufgabe sowohl mit und unter den Studierenden als auch mit und unter den Lehrenden unseres Instituts und zwischen den Statusgruppen. Um dieser Aufgabe gerecht zu werden, teilten wir das Projekt in drei wesentliche Projektbausteine ein: (1) Kommunikation, (2) Prozessmoderation und (3) Begleitforschung.

\section{(1) Kommunikation}

Unser Projekt hat an unterschiedlichen Schnittstellen eine vermittelnde Funktion. So tragen wir das Leitbild der Universitätsleitung und die Arbeitsergebnisse verschiedener Gremien im Bereich des Qualitätsmanagements für Lehre (kurz: QM) in unseren Fachbereich und unseren Studiengang sowie in die Gestaltung der einzelnen Veranstaltungen. Entsprechend koppeln wir die Aktivitäten, Veränderungen und Erfahrungen damit über das QM und die Beteiligung an zentralen Aktivitäten wieder an den Fachbereich und das Konrektorat und Referat für Lehre und Studium zurück. An unserem Institut fördern wir die Kommunikation zwischen der Leitung, 
der Studiengangkoordination und den beteiligten Lehrenden und Studierenden. Dies geschieht beispielsweise, indem wir Klausurtagungen organisieren oder Arbeitstreffen initiieren, die sich thematisch dem Forschenden Lernen und dem Umgang mit Diversität widmen. Darüber hinaus haben wir einen Lehrenden- und Studierendenrat etabliert. Diese unterstützen uns bei der Kommunikation unserer Anliegen und bei der Einbettung der Maßnahmen.

\section{(2) Prozessmoderation}

Formate wie die jährlich stattfindende Klausurtagung haben wir eingerichtet, um konzentriert mit dem gesamten Team an Innovationen des Forschenden Lernens, an der Studiengangentwicklung und an den intermodularen Verbindungen gemäß Zürcher Framework arbeiten zu können. Die in Übereinkunft gesteckten Ziele werden im Anschluss daran im Institutsalltag so weit wie möglich vorangetrieben. Wichtige Elemente für die praktische, kollegiale Arbeit am Curriculum sind die Kommunikationskultur am Institut und die wöchentlich stattfindenden Sitzungen der Lehrenden zusammen mit den Sekretariatskräften. Strukturell gilt es, das Forschende Lernen mit allen Themen rund um Lehre und Studium in Verbindung $\mathrm{zu}$ bringen, seien es Bewertungskriterien, Prüfungsformen und -bestimmungen, Feedbackkulturen oder Lehrplanungen. Forschendes Lernen wird dadurch zu einem Dauerthema im Institutsalltag. Besonders förderlich für diese Habitualisierungen ist es, wenn auch die Institutsleitung dem Forschenden Lernen einen wichtigen Stellenwert einräumt.

\section{(3) Begleitforschung}

Unser Projekt betreibt forschungsbasierte Studiengangsentwicklung, die darauf abzielt, die Curriculumsplanungen der Lehrenden mit dem Curriculumserleben der Studierenden abzugleichen. Dabei geht es darum, mehr darüber zu erfahren, wie und wo die Studierenden Elemente des Forschenden Lernens in ihrem Studienalltag erleben, ob sie den Verlauf des Studiums nachvollziehbar finden respektive selbst mitgestalten und ob sie Verbindungen zwischen den Modulen erkennen oder selbst schaffen können. Die Diversität zwischen und unter den Studierenden und Lehrenden, vor allem in Bezug auf Studienerwartungen und -erleben einerseits und Studienplanungen und -erleben andererseits, ist hierbei von hoher Bedeutung. Dieser Projektbaustein wird im Folgenden detailliert vorgestellt. 


\section{$4 \quad$ Begleitforschung als zentrales Element der Studiengangsprofilbildung}

Die Begleitforschung des Projektes verfolgt das Ziel, fundierte Kenntnisse darüber zu erlangen, wie Studierende das Forschende Lernen und im Speziellen dessen intermodulare Verknüpfungen im Studienverlauf erleben. Die Ergebnisse der Begleitforschung dienen im Abgleich mit den Modul- und Veranstaltungsplanungen der Lehrenden als Orientierung für zukünftige Schritte der Studiengangsgestaltung und sind somit eine wichtige Entscheidungsgrundlage für die systematische Umsetzung des Forschenden Lernens über den gesamten Studienverlauf. In diesem Sinne kann die Begleitforschung als eine unserer Bestrebungen aufgefasst werden, den roten Faden im Studienverlauf zu verdeutlichen und den Studiengang so für aktuelle und zukünftige Studierende noch attraktiver zu gestalten. Sinn und Zweck der Begleitforschung ist, zu erkunden, wie Studierende den Studiengang und den Aufbau des Curriculums erleben. Ihre Sicht auf Prozesse des Forschenden Lernens und auf intermodulare Verbindungen ist für eine empirisch basierte curriculare Implementierung des Forschenden Lernens grundlegend.

Dafür fokussierte die Begleitforschung auf eine Mikro- und eine Makroebene des Forschenden Lernens. Auf der Mikroebene nahmen wir ausgewählte kleinteilige Forschungsprozessschritte in Kontexten des Forschenden Lernens in den Blick. Zu deren Auswahl glichen wir die Phasen des Forschenden Lernens (Huber, 2013, S. 248) mit den Phasen des kulturwissenschaftlich-ethnografischen Forschenden Lernens (Kaufmann, 2013, S. 131-135) ab und konzentrierten uns dann auf jene Schritte, die uns für die Seminargestaltung im Sinne des Forschenden Lernens aufgrund unserer Lehrerfahrungen im Team als besonders relevant und hinsichtlich der Kenntnisse über studentische Erfahrungen und Erlebnisse als besonders aufschlussreich erschienen. Da die Begleitforschung bei den Mikroprozessen des Forschenden Lernens im Einzelmodul das Verständnis für die Perspektive der Studierenden beim Erleben des Forschungsprozesses erhöhen wollte, wurde Wert darauf gelegt, das Sprechen und Schreiben der Studierenden über ihre Arbeitsschritte in ihren Worten festzuhalten und zu reflektieren (Eisold et al., 2017). In einer Evaluation auf Veranstaltungsebene wird deutlich, dass die Studierenden das Forschende Lernen teilweise auf eine Art und Weise für ihr Studium nutzen, wie wir es so (noch) nicht erwartet hatten. Die Ergebnisse zeigen, dass die meisten unsere Bestrebungen, die Module des Studiengangs besser miteinander zu verknüpfen, teilen und ihren Studienverlauf selbst so gestalten, dass sie Verbindungen herstellen. Aus ihrer Perspektive handelt es sich dabei vor allem um thematische Verbindungen wie: 
„Ich habe mich für mein jetziges Forschungsfeld entschieden, weil ich im letzten Semester bereits ein Essay über ein verwandtes Thema geschrieben hatte. Ich fand das Thema weiterhin spannend und wollte mehr darüber erfahren."

Für die Datenerhebung zu der Makroebene des Forschenden Lernens bot es sich an, retrospektive Interviews über das Erleben des gesamten Studienverlaufs durchzuführen. Dazu haben wir weit fortgeschrittene Studierende gebeten, ihren individuellen Studienverlauf mit Schwerpunkt auf ihren konkreten Forschungserfahrungen zu rekonstruieren. Entsprechend unserer Bestrebung, eine vielfältige Auswahl an teilnehmenden Studierenden zu treffen, fällt bei der Analyse des Materials besonders auf, wie divers bereits die Studienerwartungen waren und wie unterschiedlich ein und derselbe Studiengang studiert und erlebt wurde. Nichtsdestotrotz gibt es Tendenzen, die sich über alle Interviews hinweg abzeichnen: Dies betrifft beispielsweise die Verknüpfung von Theorie und Empirie. So beschreiben die Studierenden, dass sie die grundlegenden Theorien des Fachs, die sie zu Beginn des Studiums kennengelernt hatten, trotz des Anspruchs auf enge Verbindungen zwischen Theorie und Empirie im späteren Studienverlauf kaum wieder aufgreifen konnten. Auch drücken einige den Wunsch nach Kenntlichmachung eines roten Fadens im Studienverlauf aus. Manche versuchen, über Verknüpfungen den Aufwand im Studium so gering wie möglich zu halten. Andere suchen selbst nach inhaltlichen Verbindungen, um sich so ein individuelles Studien- und Qualifikationsprofil zu erarbeiten. Diese Bestrebungen scheinen gerade zum Ende des Studiums hin als besonders sinnvoll erlebt zu werden. Einige empfinden die vielfältigen Angebote, aus denen sie sich selbst ihren roten Faden gestalten können, als genau richtig für sich. Für diese selbstständigen Studierenden, die recht gut wissen, was sie wollen, liegen im Wahl- und Wahlpflichtbereich des Studiengangs viele Angebote zur Spezialisierung sowie zur Mitgestaltung vor. Sie können gut mit dem Selbstverständnis von komplexen, vielfältigen und dynamischen kulturwissenschaftlichen Fachkulturen umgehen, die keinen engen Kanon an prüfbaren Grundlagen vorgeben, sondern Gestaltungsfreiräume und Spezialisierungen ermöglichen. Jene Studierende hingegen, die eher unsicher sind und nicht genau wissen, was sie brauchen, fordern mehr Struktur und Erläuterungen.

Abweichend von der Begleitforschung zur Mikroebene des Forschenden Lernens in einem Modul, das dieses explizit und reflektierend durchführt, machten uns die retrospektiven Interviews deutlich, dass Studierende oftmals Forschendes Lernen praktizieren, ohne es selbst als solches aufzufassen oder zu beschreiben. Der Begriff des Forschenden Lernens wird also hauptsächlich mit Modulen in Verbindung gebracht, in denen sich Studierende einer Fragestellung mit selbsterhobenem Datenmaterial annähern und möglichst einen ganzen Forschungsprozess 
durchlaufen. Je fragmentierter der Forschungsprozess hingegen ist, den die Studierenden bearbeiten oder erfahren, desto weniger bringen sie ihre Erfahrungen mit der Idee des Forschenden Lernens in Verbindung. Dies weist darauf hin, dass das „Zürcher Framework“ (Tremp \& Hildbrand, 2012) zwar den Lehrenden ein Begriff sein mag, die Verbindungen den Studierenden hingegen kaum bewusst sind und im Verlauf des Curriculums besser vermittelt werden sollten. Für die Module und Einzelveranstaltungen ist es wichtig, den Bezug zum Gesamtcurriculum zu verdeutlichen. Aus den Ergebnissen der Begleitforschung haben wir Thesen formuliert, um diese mit den Lehrenden zu diskutieren und in der Folge diverse mögliche Studierendenerfahrungen bei den Planungen mitdenken zu können. Die Arbeit am Curriculum mit den Lehrenden stellen wir im nächsten Abschnitt vor.

\section{$5 \quad$ Die Arbeit mit den Lehrenden am Curriculum}

Die Arbeit an der Profilbildung zum Forschenden Lernen erfolgt möglichst mit allen Lehrenden. Sie findet am Institut für Ethnologie und Kulturwissenschaft im Rahmen der jährlich stattfindenden zweitägigen Klausurtagungen statt sowie beim Austausch zwischen Lehrenden und Studierenden am Tag der Lehre, in Team-Kolloquien zur Verständigung über die Lehre und in den wöchentlich stattfindenden Teamsitzungen. Da sich im Fall unseres Studiengangs eher die Moduleinheiten als zentral für die Arbeit am Curriculum anbieten und weniger die Einzelveranstaltungen, wie es das Zürcher Framework vorschlägt, sind für unseren Profilbildungsprozess die Modulverantwortlichen besonders wichtig. Sie verantworten unter anderem die Modulpläne und -beschreibungen sowie die Qualitätssicherung und sie entscheiden auch gemeinsam mit der Studiengangleitung über die Gestaltung und Veränderung der Module. Grundlegend für diese Veränderungen sind in der Regel die Veranstaltungs- und Modulevaluationen, die jedoch weitgehend auf Einzelmodule beschränkt bleiben und nicht das ganze Curriculum in den Blick nehmen. Unser Projekt erweitert diese Perspektive, indem es über die Ergebnisse unserer Begleitforschung mit den Studierenden - hier vor allem die retrospektiven Interviews in der Abschlussphase - die Einzelmodule aus der Perspektive des gesamten Curriculums betrachtet. Die Aussagen zum Erleben des Studienverlaufs und zu den Zusammenhängen zwischen dessen einzelnen Elementen enthalten also aufschlussreiche Sichtweisen auf das Curriculum, die den Lehrenden gemeinhin kaum zugänglich sind. Der Forschungsfokus auf die Studienerfahrungen unterstützt zudem den Perspektivwechsel der Lehrenden in Richtung Studierendenorientierung, was für Forschendes Lernen grundlegend ist. 
Die Arbeit am Curriculum erfolgt auf der diachronischen und der synchronischen Ebene, die sich wechselseitig ergänzen. Die diachronische Ebene bezieht sich auf Verbindungen zwischen den Modulen/Einzelveranstaltungen im Studienverlauf und die synchronische auf Verbindungen zwischen gleichzeitig stattfindenden Modulen/ Veranstaltungen. Um das Forschende Lernen systematisch im Curriculum zu verankern, ordnen wir die Studierendenaktivitäten so, dass sie im curricularen Verlauf auf diachronischer Ebene sinnvoll aufeinander aufbauen. Das gesamte Curriculum lässt sich dann - einem kulturwissenschaftlich-ethnologischen Forschungsprozess mit rekursivem Design analog, das verschiedene Forschungsschritte wiederholt auf sich selbst anwendet (Breidenstein et al., 2013) - als spiralförmiges, zirkuläres Modell mit rekursiven Schleifen beschreiben. Es ordnet sich dem „Spiralcurriculum“ (siehe Huber in diesem Sammelband) zu, bei dem sich Forschendes Lernen vor allem bezogen auf den Erwerb von Forschungs- und Praxismethoden intracurricular in mehreren aufeinander aufbauenden Schleifen wiederholt. Dabei nimmt die Komplexität von den Grundlagenmodulen über die Spezialisierungsmodule bis zu den selbst gestaltbaren Modulen zu, da es gerade eine Besonderheit des Studiengangs ist, dass er diverse Wahl- und Wahlpflichtoptionen offenhält. Der bestehende Studiengang, der insbesondere über das Forschende Lernen die Forschungs- und Praxisorientierung verbindet (Kaufmann, 2015), hat dafür bereits ein passendes Studiengangsmodell etabliert, das im ersten Jahr Grundlagenmodule konzipiert, im zweiten Jahr die Methodenausbildung sowie Aufbau-/Vertiefungsmodule und im dritten Jahr spezifische Schwerpunktmodule sowie ein Praxis-, ein Selbststudienund das Abschlussmodul. Im curricularen Verlauf lernen die Studierenden in den Grundlagenmodulen von Beginn an kulturwissenschaftliche Fragestellungen, den Stand der Forschung, zentrale Begriffe und Theorien sowie einzelne Prozessschritte fächerspezifischen Forschens kennen und üben sich in kulturwissenschaftlichen Arbeitstechniken. Sie führen hier bereits kleine teilnehmende Beobachtungen durch. In den intensiven Methodenmodulen forschen sie im Rahmen von Studienforschungen selbst und machen dabei die Erfahrung sämtlicher Forschungsschritte (Huber, 2009; Kaufmann, 2013, 2018). Dabei beginnen sie mit der Entwicklung einer eigenen Fragestellung, verfassen abschließend einen Forschungsbericht und veröffentlichen diesen nach Möglichkeit im Rahmen unserer Studierendentagung „ResearchInsights“. Im späteren Studienverlauf wiederholen sie das gelernte empirische Arbeiten auf höherem Niveau im Rahmen eines Selbststudienmoduls mittels einer eigenständigen Forschungsarbeit. Hier arbeiten sie ohne methodische Anleitungen, oftmals in Form einer thematischen oder methodischen Vorstudie für die Bachelorarbeit, und schließen den Bachelor im Abschlussmodul mit einem eigenständigen Forschungsprojekt ab. 
Dieses Modell des Studiengangs haben wir durch die Begleitforschung und die Arbeit mit den Lehrenden expliziert. Nun gilt es, dies auch für die Studierenden deutlicher zu kommunizieren, um ihnen den roten Faden im Studienverlauf zu verdeutlichen, den sie zum Teil vermissten. Dabei erlangt durch unser Projekt auch die synchronische Ebene eine hohe Bedeutung. Hierbei geht es um eine engere Verknüpfung von Modulen und Veranstaltungen, die zeitgleich stattfinden, wodurch sich neue Synergien ergeben können. Inzwischen haben wir beispielsweise begonnen, das in Gänze mit Forschendem Lernen durchgeführte Methodengrundlagenmodul mit dem gleichzeitig stattfindenden Theoriemodul systematisch zu verbinden. Dafür stehen nun die Lehrenden beider Module im Austausch miteinander und unterstützen die Studierenden darin, unsere Verbindungsangebote zu erproben. Damit können wir für beide Module Verbesserungen erzielen, da ansonsten im Methodenmodul nicht genug Zeit bleibt, ausreichend an den Theorieeinbettungen zu arbeiten, und die Arbeit im Theoriemodul so durch die Forschungspraxis konkretisiert wird. Durch solche intermodularen Verknüpfungen können wir das Forschende Lernen im Studiengangsprofil intensivieren. Unsere Aufgabe bestand also auch darin, die Modulverantwortlichen ins Gespräch miteinander zu bringen, damit sie untereinander zu den Modulinhalten kommunizieren, an den Verbindungen arbeiten und diese in der Folge auch den Studierenden besser erklären können. Sie suchen nun gezielter nach Verbindungen zwischen einzelnen Seminaren und können durch ihre Kenntnisse der Pläne und Durchführungen der anderen Lehrenden den Studierenden eher Hinweise auf mögliche weitere Verknüpfungen zwischen den Modulen geben. Je besser sämtliche Lehrenden über das Gesamtgeschehen informiert sind, desto weniger bedarf es der zusätzlichen Studienberatung. Auch dies bestätigt die zentrale Bedeutung der Kommunikation und Kollaboration mit und unter den unterschiedlichen Beteiligten für die Profilbildung.

Um dem Profil Gewicht zu geben, haben wir zum Projektende mit Lehrenden und Studierenden ein Faltblatt zum Studiengang erstellt, das sich an (potenzielle) Studierende richtet und den Studiengang auch für Außenstehende auf einfache Weise veranschaulicht.

\section{$6 \quad$ Zentrale Elemente der Implementierung}

Unser Projekt ist ein Beispiel für die intracurriculare Verankerung des Forschenden Lernens im Rahmen eines Spiralcurriculums (siehe Huber in diesem Sammelband). Die Implementierung wurde als Entwicklungsprozess mit dem gesamten Institutsteam und in Zusammenarbeit mit Studierenden durchgeführt. Dabei hat es sich 
für uns als sinnvoll herausgestellt, die Lehrgestaltung mit den Studienerfahrungen abzugleichen und im Anschluss daran gegebenenfalls zu modifizieren. Dies setzt voraus, dass die Lehrenden untereinander und mit den Studierenden kommunizieren und kollaborieren.

Das beschriebene Projekt hat uns ermöglicht, die hierfür notwendige Moderation zu übernehmen. Die Implementierung erfolgte forschungsbasiert. Bei der Begleitforschung war es von Vorteil, Methoden zu wählen, die in unserem Fach praktiziert werden. Dies erhöht die Wahrscheinlichkeit, dass auch die Forschungsergebnisse und die abgeleiteten Handlungsempfehlungen akzeptiert werden. All diese Ebenen - der Einbezug der Lehrenden und Studierenden, die Beachtung der zentralen Bedeutung der Kommunikation, die Prozessmoderation durch dafür bestimmte Personen sowie die Formen der Evaluation und der Begleitforschung können wir für die Implementierung in anderen Studiengängen weiterempfehlen.

Schwierigkeiten ergaben sich jedoch beispielsweise daraus, dass die Implementierung ein sehr langwieriger Prozess ist. Allein schon das Ziel, in einem größeren Lehrteam zu einem gemeinsamen Verständnis des Forschenden Lernens zu kommen, braucht Geduld. Noch immer empfinden uns einige der Lehrenden als „dogmatisch“, weil wir auf den Definitionen des Forschenden Lernens von Ludwig Huber beharren. Auch bei der Feinarbeit in der Umsetzung gibt es Hürden zu überwinden, da das Konzept nicht allen Lehrenden und Studierenden gleichermaßen entgegenkommt. Schwierigkeiten bereiten darüber hinaus die Überlastung der Lehrenden und die hierarchischen Strukturen von Universitäten. Auch die mangelnden Ressourcen für das Forschende Lernen - wie der Mangel an geeigneten Räumen, zu große Lerngruppen und die prekären Stellen einiger Lehrender - erschweren unsere Arbeit zum Forschenden Lernen als Studiengangsprofil.

Um curriculare Modelle des Forschenden Lernens zu entwickeln, in denen das Studium als längerer Forschungsprozess gedacht wird, ist auf zirkuläre, rekursive Schleifen - Spiralmodelle - zu achten. Dabei ist bei der Verbindung der Einzelmodule auf diachronischer Ebene der rote Faden im Studienverlauf hervorzuheben. Er ist nicht nur für die Planungen und Durchführungen der Lehrenden wichtig, sondern auch für die Orientierung der Studierenden. Auf synchronischer Ebene lassen sich durch intermodulare Verknüpfungen Synergien erzeugen. Bei der Profilbildung des Studiengangs ist aus unserer Sicht stets darauf zu achten, dass sie nicht auf ein einzig mögliches curriculares Modell des Forschenden Lernens abzielen, sondern verschiedene, fachspezifische Varianten eröffnen. 


\section{Literatur}

Breidenstein, G., Hirschauer, S., Kalthoff, H. \& Nieswand, B. (2013). Ethnografie: Die Praxis der Feldforschung. Konstanz: UVK.

Eisold, L., Kaufmann, M. E., Koch, H. \& Tiepermann, I. (2017). „Wir befinden uns in ungewohnten Situationen und lernen daraus" - über Erkenntnismomente beim Forschenden Lernen. Resonanz - Magazin für Studium und Lehre an der Universität Bremen, Sommersemester 17, S. 27-32.

Ghaffarizad, K., Kaufmann, M. E., Koch, H., Reuter, A., Kurzawski, B. \& Seufert, P. (2015). Forschendes Lernen als Teamplay: Gemeinsamer Bericht von Studierenden und Lehrenden über den Tag der Lehre 2014. Resonanz - Magazin für Studium und Lehre an der Universität Bremen, 3, S. 9-14.

Huber, L. (2009). Warum Forschendes Lernen nötig und möglich ist. In L. Huber, J. Helmer \& F. Schneider (Hrsg.), Forschendes Lernen im Studium: Aktuelle Konzepte und Erfahrungen (S. 9-35). Bielefeld: UVW.

Huber, L. (2013). Methodische Anregungen für den Umgang mit pragmatischen Schwierigkeiten im Forschenden Lernen. In L. Huber, M. Kröger \& H. Schelhowe (Hrsg.), Forschendes Lernen als Profilmerkmal einer Universität: Beispiele aus der Universität Bremen (S. 247-255). Bielefeld: UVW.

Huber, L., Kröger, M. \& Schelhowe, H. (Hrsg.) (2013). Forschendes Lernen als Profilmerkmal einer Universität: Beispiele aus der Universität Bremen. Bielefeld: UVW.

Kaufmann, M. E. (2013). „Wir haben selbst neue Wissenszusammenhänge geschaffen!“ Forschendes Lernen zu „Diversity“ in einer Großveranstaltung zur Methodenlehre im BA-Studiengang Kulturwissenschaft. In L. Huber, M. Kröger \& H. Schelhowe (Hrsg.), Forschendes Lernen als Profilmerkmal einer Universität: Beispiele aus der Universität Bremen (S. 123-142). Bielefeld: UVW.

Kaufmann, M. E. (2015). Forschendes Lernen als Bindeglied zwischen Forschungs- und Berufsorientierung in geisteswissenschaftlichen Studiengängen. In P. Tremp (Hrsg.), Forschungsorientierung und Berufsbezug im Studium: Blickpunkt Hochschuldidaktik (S. 151-170). Bielefeld: Bertelsmann.

Kaufmann, M. E. (2018). Communities of Practice: Forschendes Lernen in Ethnologie und Kulturwissenschaft. In M. E. Kaufmann, A. Satilmis \& H. A. Mieg (Hrsg.), Forschendes Lernen in den Geisteswissenschaften: Konzepte, Praktiken und Perspektiven hermeneutischer Fächer (S. 169-190). Wiesbaden: Springer VS.

Kaufmann, M. E. \& Koch, H. (2015a). Die Lehrenden als Kompliz_innen im forschenden Lernprozess. In K. Rheinländer (Hrsg.), Ungleichheitssensible Hochschullehre (S. 219-236). Wiesbaden: Springer VS.

Kaufmann, M. E. \& Koch, H. (2015b). Evaluierende Begleitforschung zum Umgang mit Heterogenität beim Forschenden Lernen in der Studieneingangsphase. In S. Harris-Huemmert, L. Mitterauer \& P. Pohlenz (Hrsg.), Heterogenität der Studierenden: Herausforderung für die Qualitätsentwicklung in Lehre und Studium, neuer Fokus für die Evaluation? (S. 113-128) Bielefeld: UVW.

Kaufmann, M. E. \& Koch, H. (2018). Forschendes Lernen in der curricularen Profilbildung. In M. E. Kaufman, A. Satilmis \& H. A. Mieg (Hrsg.), Forschendes Lernen in den Geisteswissenschaften: Konzepte, Praktiken und Perspektiven hermeneutischer Fächer (S.79-102). Wiesbaden: Springer VS. 
Kaufmann, M. E. \& Schelhowe, H. (2017). Forschendes Lernen als Lehrprofil von Hochschulen - am Beispiel der Universität Bremen. In H. A. Mieg \& J. Lehmann (Hrsg.), Forschendes Lernen: Wie die Lehre in Universität und Fachhochschule erneuert werden kann (S.392-400). Frankfurt: Campus.

Satilmis, A. \& Voß, M. (2017). „Exploring Diversity!“ - Diversität und Forschendes Lernen entdecken und gestalten: Eine inter- und transdisziplinäre Kooperation. Resonanz Magazin für Lehre und Studium der Universität Bremen, Sommersemester 2017, S. 33-40.

Tremp, P. \& Hildbrand, T. (2012). Forschungsorientiertes Studium - universitäre Lehre: Das „Zürcher Framework“ zur Verknüpfung von Lehre und Forschung. In T. Brinker \& P. Tremp (Hrsg.), Einführung in die Studiengangentwicklung (S. 101-116). Bielefeld: Bertelsmann.

\section{Weitere Quellen}

Universität Bremen (Hrsg.) (2015). Leitbild für Lehre und Studium an der Universität Bremen (16.12.2015). Verfügbar unter https://www.uni-bremen.de/studium/lehre-studium/ leitbild [30.08.2018].

Strategiedokument der Universität Bremen (2018). Verfügbar unter https://www.uni-bremen. de/fileadmin/user_upload/universitaet/Strategiedokument/Strategie_2018-2028_Universitaet_Bremen.pdf [20.02.2020]].

\section{Zur Autorin und zum Autor}

Margrit E. Kaufmann, Dr. phil., ist Senior Researcher für Ethnologie und Kulturwissenschaft am Fachbereich 9 der Universität Bremen. Als Expertin für Diversity berät und begleitet sie Organisationsprozesse und gestaltet Projekte zu Forschendem Lernen und Diversity. Ihre Lehr- und Forschungsschwerpunkte sind: Intersektionelle Diversity Studies \& Cultural Complexity, Flucht \& Asyl, Organisationsforschungen, Forschendes Lernen, Methoden und Theorie-Praxis-Transfer. E-Mail: mkaufm@ uni-bremen.de

Henning Koch, M. A., war Wissenschaftlicher Mitarbeiter am Institut für Ethnologie und Kulturwissenschaft der Universität Bremen. Hier führte er das Projekt „Forschendes Lernen als Studiengangsprofil “ durch. In seiner Dissertation beschäftigt er sich mit juristischen Fachkulturen und der Frage, wie in ihnen etablierte und außenstehende Studierende konstruiert werden. Seine Forschungsschwerpunkte sind: Hochschulforschung, Bildungsforschung, Qualitative Methoden in der Hochschulforschung, Intersektionelle Diversity Studies, Forschendes Lernen, Hochschuldidaktik. E-Mail: henning.koch@uni-bremen.de 
Open Access Dieses Kapitel wird unter der Creative Commons Namensnennung 4.0 International Lizenz (http://creativecommons.org/licenses/by/4.0/deed.de) veröffentlicht, welche die Nutzung, Vervielfältigung, Bearbeitung, Verbreitung und Wiedergabe in jeglichem Medium und Format erlaubt, sofern Sie den/die ursprünglichen Autor(en) und die Quelle ordnungsgemäß nennen, einen Link zur Creative Commons Lizenz beifügen und angeben, ob Änderungen vorgenommen wurden.

Die in diesem Kapitel enthaltenen Bilder und sonstiges Drittmaterial unterliegen ebenfalls der genannten Creative Commons Lizenz, sofern sich aus der Abbildungslegende nichts anderes ergibt. Sofern das betreffende Material nicht unter der genannten Creative Commons Lizenz steht und die betreffende Handlung nicht nach gesetzlichen Vorschriften erlaubt ist, ist für die oben aufgeführten Weiterverwendungen des Materials die Einwilligung des jeweiligen Rechteinhabers einzuholen.

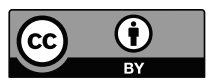

